\title{
LÍNEAS MANTENEDORAS (LÍNEAS B) Y RESTAURADORAS (LÍNEAS R) DE SORGO GRANÍFERO ADAPTADAS A LOS VALLES ALTOS CENTRALES DE MÉXICO
}

\author{
MAINTAINER (B_LINES) AND RESTORER (R_LINES) GRAIN SORGHUM \\ LINES ADAPTED TO THE MEXICĀN HIGHLANDS
}

\author{
Leopoldo E. Mendoza-Onofre ${ }^{1 *}$, Ma. Eugenia Cisneros-López ${ }^{2}$, Marisol \\ Galicia-Juárez ${ }^{1}$ y Miguel Hernández-Martínez ${ }^{3}$
}

\begin{abstract}
${ }^{1}$ Campus Montecillo, Colegio de Postgraduados. Km. 36.5 Carr. México-Texcoco. 56230, Montecillo, Estado de México, México. ${ }^{2}$ Campo Experimental Río Bravo, Instituto Nacional de Investigaciones Forestales, Agrícolas y Pecuarias (INIFAP). Km. 61 Carr. Matamoros-Reynosa. 88900, Tamaulipas, México. ${ }^{3}$ Campo Experimental El Bajío, INIFAP. Km. 6.5 Carr. Celaya-San Miguel de Allende. 38810, Celaya, Gto.
\end{abstract}

*Autor para correspondencia (leopoldo@colpos.mx)

Los Valles Altos Centrales de México (cerca de 1.5 millones de hectáreas) comprenden áreas agrícolas con altitudes de 1800 a 2300 m. En esta región no existen siembras comerciales de sorgo (Sorghum bicolor L. Moench), pues las variedades e híbridos disponibles no producen grano ya que las bajas temperaturas (entre 5 y $12^{\circ} \mathrm{C}$ ) que prevalecen antes de la floración provocan que el polen sea estéril (Livera y Carballo, 1985). Estas temperaturas se presentan incluso en regiones donde se cultiva comercialmente sorgo, lo que favorece la incidencia de cornezuelo (Claviceps africana) (Hernández-Martínez et al., 2002). Los esfuerzos por generar sorgos tolerantes al frío (TF) fructificaron con la liberación de tres variedades de polinización libre por el entonces Instituto Nacional de Investigaciones Agrícolas (Romo y Carballo, 1980), adoptándose como principal criterio agronómico de tolerancia al frío el que las panojas autofecundadas presentaran al menos $90 \%$ de grano. De esas variedades, solamente VA-110 se mantuvo en el mercado por pocos años.

Con el propósito de iniciar un proyecto de desarrollo de híbridos de sorgo TF, en el Colegio de Postgraduados (CP) se generaron líneas mantenedoras (líneas de respuesta B) y restauradoras (líneas de respuesta $R$ ) adaptadas a esas condiciones ecológicas (Mendoza-Onofre, 1992). El proyecto se inició en el año 1980, con 250 accesiones de India y África procedentes del Instituto Internacional de Investigación de Cultivos para las Zonas Tropicales Semiáridas (ICRISAT, por sus siglas en inglés) y donadas al CP por conducto del Centro Internacional de Mejoramiento de Maíz y Trigo (CIMMYT). Las accesiones de mejor aspecto agronómico y con mayor tolerancia al frío se cruzaron

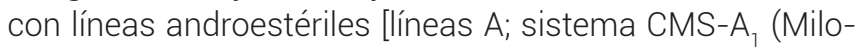
Kafir), donadas al CP por la Universidad de Nebraska, USA]. Solamente cinco accesiones presentaron respuesta mantenedora (potenciales líneas B), de las que se derivaron sus respectivas líneas $A$ isogénicas mediante al menos cinco retrocruzas. En el caso de las accesiones que presentaron respuesta restauradora, las de mejor aspecto agronómico se sometieron a varios ciclos de selección individual; en otros casos se generó nueva variabilidad mediante la aplicación de agentes mutagénicos físicos o químicos y se aplicó selección por pedigrí en las generaciones recombinantes hasta lograr la uniformidad deseada.

Al final del proceso se obtuvieron líneas A, B y $R$ tolerantes al frío, las cuales se designaron como "líneas TF de primera generación" (Osuna-Ortega et al., 2001, 2003). Con objeto de incrementar el número de líneas B, en 1986 se hicieron los cruzamientos posibles, mediante emasculaciones manuales, entre las cinco líneas $B$ iniciales y a partir de la $F_{2}$ se efectuó selección por pedigrí durante seis ciclos generándose nuevas líneas B-TF, así como sus correspondientes líneas A. En el caso de las líneas R, durante los años 1990 a 2010 se efectuaron evaluaciones de campo con fines diversos, observándose que el rendimiento de grano y las características agronómicas de tipo de planta, precocidad, sanidad, entre otras, de algunas líneas R-TF, eran mejores que las de VA-110. De esta manera se seleccionaron nuevas líneas A, B y $R$ tolerantes al frío, a las que se denominó "líneas TF de segunda generación" (León-Velasco et al., 2009).

Con estas líneas se han formado híbridos experimentales de sorgo adaptados a los Valles Altos Centrales de México (León-Velasco et al., 2009). Además, los cruzamientos entre líneas A susceptibles al frío (adaptadas a las regiones sorgueras tradicionales; i.e., Tamaulipas y El Bajío) con líneas R-TF, así como las cruzas entre las líneas A-TF con líneas $\mathrm{R}$ susceptibles al frío, producen híbridos experimentales de alto rendimiento de grano. Al respecto, en Río Bravo, Tamps., bajo riego, el rendimiento promedio de ese tipo de híbridos y los testigos VA-110, Purépecha y RB-4000 fue 3215, 2009, 1695 y $2003 \mathrm{~kg} \mathrm{ha}^{-1}$, respectivamente. En 
Celaya, Gto., también con riego, los rendimientos respectivos fueron 8253, 6005, 7189 y $6802 \mathrm{~kg} \mathrm{ha}^{-1}$. Esto significa que el uso de germoplasma tolerante al frío permite ampliar la adaptación y adaptabilidad de esta especie en México (Osuna-Ortega et al., 2001).

Como resultado de estas evaluaciones, se han seleccionado cuatro líneas mantenedoras (B1, B2, B3 y B5) y tres líneas restauradoras (R17, R19 y R25) para registrarlas en el Catálogo Nacional de Variedades Vegetales (CNVV) de México (Cuadro 1).

Las líneas B y R han mostrado buena adaptación en los Valles Altos Centrales de México. En tres localidades del Estado de México (Santa Lucía, Tecámac y Montecillo), el rendimiento de semilla promedio por panoja de las líneas $R$ fue mayor que el de las líneas B (38 g vs. 24 g) en riego, pero dicho rendimiento fue de magnitud similar para ambos grupos de líneas en secano (8 g) (León-Velasco et al., 2009). En el año 2014, en Montecillo, el rendimiento promedio de las líneas $\mathrm{R}$ osciló entre 32 y 38 g por panoja, en promedio de dos fechas de siembra (entre 15 y $25 \mathrm{~g}$ para las líneas B) (Cuadro 2). El carácter de tolerancia al frío (más de $90 \%$ de grano en panojas autofecundadas) de estas líneas ha resultado estable en evaluaciones efectuadas en Celaya, Gto., Río Bravo, Tamps. (Osuna-Ortega et al., 2001) y en varias localidades y fechas de siembra en Montecillo, México (León-Velasco et al., 2009; Mendoza-Onofre et al., 2006; Osuna-Ortega, 2001). Las líneas R producen más flores, más granos de polen y de mayor tamaño, y su periodo de floración es más largo que el de las líneas B (Cisneros-López et al., 2009).

La descripción fenotípica de estas líneas se efectuó con base en los descriptores de sorgo emitidos por la Unión Internacional para la Protección de las Obtenciones Vegetales (UPOV, 2015). La información, con excepción del peso de semilla por panoja, se obtuvo en condiciones de riego en dos localidades. La primera, en Montecillo, Edo. de México ( $19^{\circ} 29^{\prime} \mathrm{N}$ y $98^{\circ} 54^{\prime} \mathrm{O}, 2250 \mathrm{~m}$ de altitud) durante el ciclo P.-V. 2014 y la segunda en el ciclo O.-I. 2014 en Agua Blanca, Municipio de Villa Juárez, Sonora (2658' N y $109^{\circ}$ 43' O, $20 \mathrm{~m}$ de altitud). En ambos ambientes se empleó a la variedad de polinización libre VA-110 como testigo (Cuadro 2).

El tipo de planta de ambos grupos de líneas contrasta con el de VA-110 (testigo). Las líneas B y R no presentan pigmentación de antocianinas en las láminas foliares; son insensibles al fotoperiodo; la planta presenta de uno a dos macollos, los cuales se desarrollan especialmente después de la antesis; el color del follaje es verde medio; las espiguillas no presentan aristas o éstas son cortas; las glumas son largas y cubren aproximadamente $75 \%$ de la semilla; la presencia de taninos en el grano es escasa o ausente; el endospermo predominante es harinoso (75\%) y el resto es vítreo. El color del grano de las líneas B es cremoso opaco y el de las líneas $R$ varía de marrón claro (VA 610) a marrón oscuro (VA 620 y VA 630). En cambio, VA-110 tiene coleoptilo pigmentado; presenta aristas en la lema; la gluma es de color café obscuro en madurez; el color de grano es marrón rojizo, con taninos y endospermo $100 \%$ harinoso. Otras características morfológicas entre estas líneas se observan en el Cuadro 2. En la Figura 1 se ilustran algunos ejemplares representativos de las panojas.

Las líneas B y R presentan buena tolerancia al ergot (Claviceps africana) (Hernández-Martínez et al., 2002; Mendoza-Onofre et al., 2006) y al tizón de la panoja y pudrición del tallo [Fusarium verticillioides (Sacc.) Nirenberg] (Cisneros-López et al., 2007). En condiciones tropicales (Tizimín,Yucatán; $21^{\circ} 08^{\prime} \mathrm{N}$ y $88^{\circ} 09^{\prime}$ 0, 20 m de altitud), durante el ciclo O.-I. (diciembre-enero) resultaron moderadamente tolerantes a la roya (Puccinia sorghi) y en las

Cuadro 1. Denominación, genealogía y número de registro definitivo en el CNVV de líneas mantenedoras $\mathrm{B}$ y líneas $\mathrm{R}$ tolerantes al frío.

\begin{tabular}{|c|c|c|}
\hline Nombre & Genealogía & Registro definitivo \\
\hline \multicolumn{3}{|c|}{ Líneas B } \\
\hline CP-TF B1 & $\{[(\mathrm{L} 2 \mathrm{~A} \times \mathrm{BT} 100-3-1-2-1-2) \mathrm{RC}$ ] $] \times \mathrm{BTF} 1\}-$ Sel 1 & SOG-276-290916 \\
\hline CP-TF B2 & $\{[(\mathrm{L} 2 \mathrm{~A} \times \mathrm{BT} 100-2-2-2-1-3) \mathrm{RC} 1] \times \mathrm{BTF} 4\}-$ Sel 2 & SOG-277-290916 \\
\hline CP-TF B3 & $\{[($ BT100-3-1-2-1-2 × BTF15) RC3] $\times$ BTF6 $\}-$ Sel 3 & SOG-279-290916 \\
\hline CP-TF B5 & $\{[(\mathrm{BT} 110-1-1-2-4-3 \times$ BTF14) RC3] $\times$ BTF9 $\}-$ Sel 5 & SOG- 274-290916 \\
\hline \multicolumn{3}{|c|}{ Líneas R } \\
\hline VA-610 & R17: (Compuesto Dietil Sulfonato 4957) F1-1-1 Sel 17 & SOG-278-210916 \\
\hline VA-620 & R19: [(SOCB 37-4PL -1-1PL-1) grano blanco-24-26-2-2-2] Sel 19 & SOG-273-210916 \\
\hline VA-630 & R 25: [(NY666-1 × H-I-MUT 1)-2-3-1] Sel 25 & SOG-275-210916 \\
\hline
\end{tabular}


Cuadro 2. Variables morfológicas del grupo de líneas $B$ y R. Datos promedio de dos ambientes.

\begin{tabular}{|c|c|c|c|c|c|c|c|c|c|}
\hline Genotipo & $\mathrm{EP}^{+}$(días) & $\mathrm{AP}^{+}(\mathrm{cm})$ & $\mathrm{DT}^{+}(\mathrm{mm})$ & $\mathrm{LH}^{+}(\mathrm{cm})$ & $\mathrm{AH}^{+}(\mathrm{cm})$ & $\mathrm{LP}^{+}(\mathrm{cm})$ & $\mathrm{LE}^{\dagger}(\mathrm{cm})$ & $\mathrm{PMS}^{+}(\mathrm{g})$ & $\mathrm{PSP}^{++}(\mathrm{g})$ \\
\hline \multicolumn{10}{|c|}{ Líneas B } \\
\hline CP-TF B1 & 70 & 100 & 13.8 & 55 & 6.0 & 28.0 & 7.8 & 18.4 & 25.1 \\
\hline CP-TF B2 & 75 & 99 & 12.6 & 52 & 6.0 & 23.1 & 5.3 & 19.5 & 18.3 \\
\hline CP-TF B3 & 64 & 95 & 14.2 & 54 & 6.2 & 26.4 & 4.1 & 23.8 & 15.2 \\
\hline CP-TF B5 & 68 & 100 & 11.8 & 50 & 4.8 & 23.5 & 12.1 & 23.1 & 15.5 \\
\hline \multicolumn{10}{|c|}{ Líneas R } \\
\hline VA 610 & 79 & 152 & 18.0 & 61 & 6.4 & 23.5 & 5.5 & 36.2 & 31.7 \\
\hline VA 620 & 81 & 125 & 17.6 & 61 & 6.7 & 26.2 & 6.1 & 33.2 & 32.1 \\
\hline VA 630 & 82 & 132 & 15.0 & 48 & 6.0 & 22.8 & 5.5 & 27.7 & 37.3 \\
\hline \multicolumn{10}{|c|}{ Testigo } \\
\hline VA-110 & 86 & 101 & 15.6 & 54 & 5.4 & 19.2 & 2.3 & 19.4 & 18.5 \\
\hline
\end{tabular}

EP. emergencia de la panoja; AP. altura de planta; DT: diámetro de tallo; LH: largo de la $3^{a}$ hoja; AH: ancho de la 3a hoja; LP. longitud de la panoja; LE: Iongitud de excersión; PMS: peso de mil semillas; PSP. peso de semilla por panoja. `En estas variables los dos ambientes fueron: Agua Blanca Municipio de Villa Juárez, Sonora (ciclo O.-I. - 2014) y Montecillo, Edo. de México (ciclo P.-V. - 2014). ${ }^{\dagger+}$ Para PSP los dos ambientes fueron dos fechas de siembra en el ciclo P.-V. 2014, en Montecillo, Edo. de México.
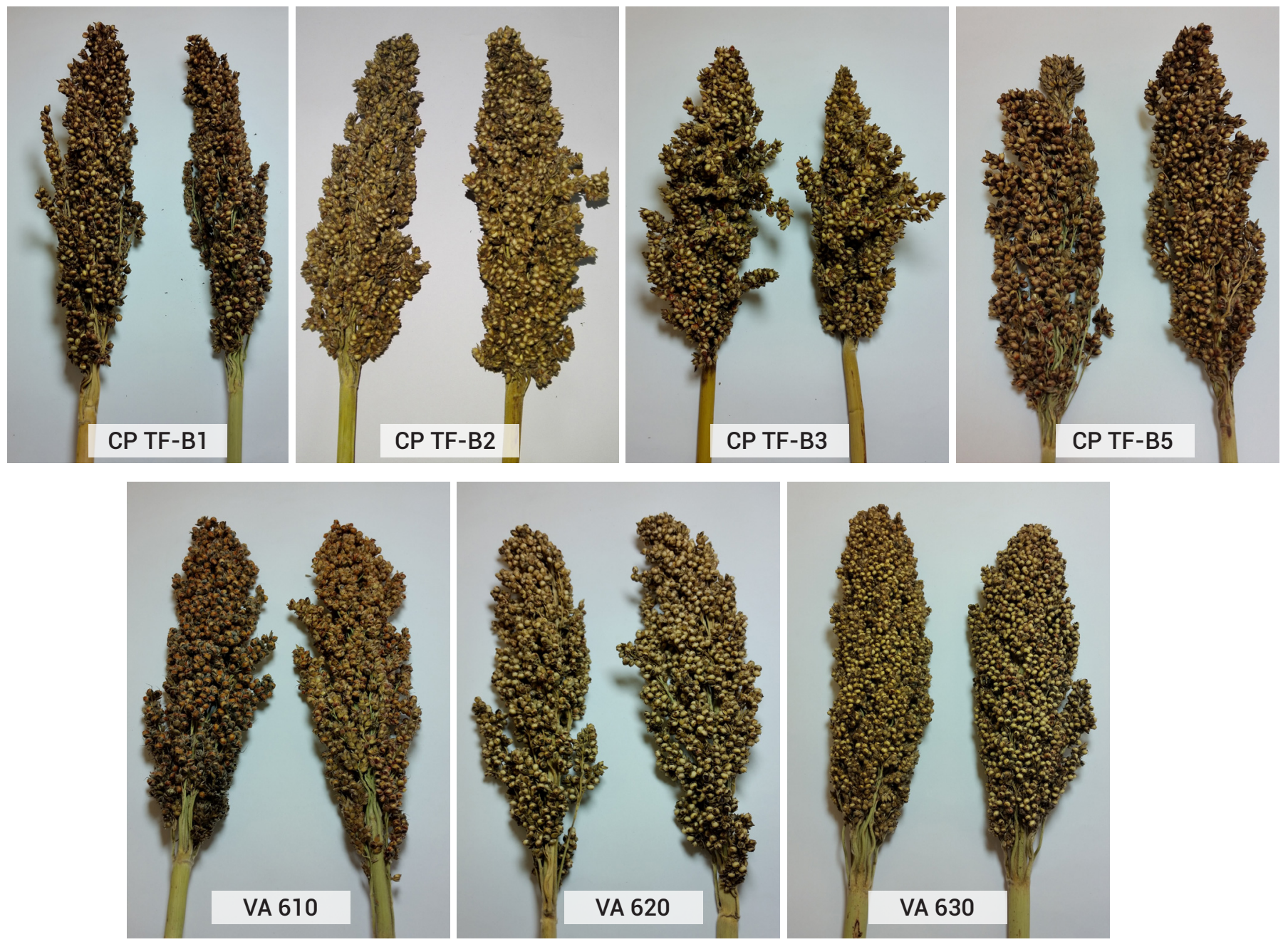

Figura 1. Panojas representativas de las líneas mantenedoras y restauradoras de sorgo adaptadas a los Valles Altos Centrales de México. 
siembras de P.-V. (mayo-junio) fueron susceptibles a la antracnosis (Colletotrichum graminicola).

La semilla original de las líneas está a disposición de los interesados en el Campus Montecillo del Colegio de Posgraduados, Montecillo, Edo. de México.

\section{AGRADECIMIENTOS}

Se agradece el apoyo de los estudiantes becarios mexicanos del CONACYT, que han participado en este proyecto, así como del M. C. Julián Barrera Sánchez y su equipo de trabajo por las facilidades brindadas para completar la descripción varietal.

\section{BIBLIOGRAFÍA}

Cisneros-López M. E., L. E. Mendoza-Onofre, G. Mora-Aguilera, L. CórdovaTéllez y M. Livera-Muñoz (2007) Híbridos y progenitores de sorgo tolerantes al frío. I: Calidad de la semilla y su influencia en el establecimiento de plántulas. Agrociencia 41:45-55.

Cisneros-López M. E., L. E. Mendoza-Onofre, H. A. Zavaleta-Mancera, V. A. González-Hernández, L. Córdova-Téllez, M. Hernández-Martínez and G. Mora-Aguilera (2009) Floral traits and seed production of sorghum $A_{-}, B_{-}$and $R_{-}$lines under chilling field temperatures. Journal of Agronomy and Crop Science 195:464-471.

Hernández-Martínez M., L. E. Mendoza-Onofre, P. Ramírez-Vallejo, S. OsadaKawasoe, E. Cárdenas-Soriano and F. Zavala-García (2002) Response of sorghum $B$ and $R$ lines to ergot (Claviceps africana) at Cela- ya, Guanajuato, México. In: Sorghum and Millets Diseases. J. F. Leslie (ed.). Iowa State Press. Ames, lowa, USA. pp:83-85.

Livera M. M. y A. Carballo C. (1985) Ampliación de las áreas de adaptación del sorgo (Sorghum bicolor L. Moench). I: Análisis del potencial productivo de genotipos tolerantes al frío en los Valles Altos. Fitotecnia 7:96-113.

León-Velasco H., L. E. Mendoza-Onofre, F. Castillo-González, T. CervantesSantana y A. Martínez-Garza (2009) Evaluación de dos generaciones de híbridos y progenitores de sorgo tolerantes al frío. II: aptitud combinatoria, heterosis y heterobeltiosis. Agrociencia 43:609-623

Mendoza-Onofre L. E. (1992) Grain yield of the first cold tolerant sorghum hybrids developed in Mexico. Sorghum Newsletter 33:62.

Mendoza-Onofre L. E., M. Hernández-Martínez, E. Cárdenas-Soriano y P. Ramírez-Vallejo (2006) El germoplasma de sorgo tolerante al frío como fuente potencial de tolerancia al ergot (Claviceps africana Frederickson, Mantle \& de Milliano). Agrociencia 40:593-603.

Osuna-Ortega J., M. C. Mendoza-Castillo and L. E. Mendoza-Onofre (2003) Sorghum cold tolerance, pollen production, and seed yield in the Central High Valleys of Mexico. Maydica 48:125-132.

Osuna-Ortega J., L. E. Mendoza-Onofre, F. Castillo-González, V. A. GonzálezHernández, M. C. Mendoza-Castillo, H. Williams-Alanís y M. Hernández-Martínez (2001) Potencial del germoplasma tolerante al frío en la adaptación y adaptabilidad del sorgo granífero en México: II. Río Bravo, Tamaulipas; y Celaya, Guanajuato. Agrociencia 35:625-636.

Romo C. E. y A. Carballo C. (1980) Características de tres variedades de sorgo para los Valles Altos. Circular CIAMEC 130. INIA-SARH. Chapingo, México. 11 p.

UPOV, Unión Internacional para la Protección de las Obtenciones Vegetales (2015) Sorgo. Directrices para la ejecución del examen de la distinción, la homogeneidad y la estabilidad. Documento TG/122/4. Unión Internacional para la Protección de las Obtenciones Vegetales. Ginebra. 35 p. http://www.upov.int/edocs/ tgdocs/es/tg122.pdf. (Septiembre 2015). 